\title{
Microscopy and Microanalysis of Mineral Particles in Lung Biopsies of Iraq and Afghanistan Deployers, Normal Controls, and Autoimmune Lung Disease Controls
}

\author{
H. Lowers ${ }^{1}$, T. Todorov ${ }^{1}$, G. Breit ${ }^{1}$, G.P. Meeker ${ }^{1}$, G.S. Plumlee ${ }^{1}$, M. Robinson ${ }^{2,3}$, R. Meehan ${ }^{2,3}$,

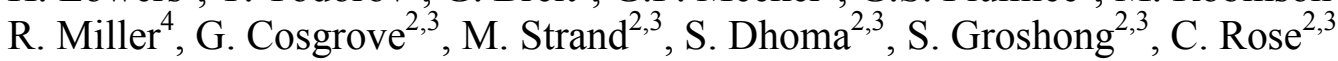 \\ ${ }^{1}$ U.S. Geological Survey, Denver, CO, USA \\ ${ }^{2}$ National Jewish Health, Denver, CO, USA \\ ${ }^{3}$ University of Colorado Anschutz Medical Campus, Aurora, CO, USA \\ ${ }^{4}$ Vanderbilt University, Nashville, TN, USA
}

US military personnel deployed to Iraq and Afghanistan have been variably exposed to desert dusts, smoke from burn pits, and urban particulate matter, and some have developed constrictive bronchiolitis. We performed FE-SEM/EDS and LA-ICP-MS analyses as part of a larger blinded study to identify the particle types present, count numbers of particles of different types, and map the distribution of particles and metals in biopsied lung tissue sections collected from deployers, a normal age- and smokingmatched control group, and an autoimmune control group.

Serial sections, $5 \mu \mathrm{m}$ and $20 \mu \mathrm{m}$ thick, of paraffin-fixed biopsied lung tissue were cut with a microtome to prepare hematoxylin and eosin (H\&E) slides $(5 \mu \mathrm{m})$, FE-SEM slices on carbon planchettes $(20 \mu \mathrm{m})$, and LA-ICP-MS slices $(20 \mu \mathrm{m})$ on high purity quartz slides. From the H\&E slides, a pulmonary pathologist identified areas of interest including those: representing normal tissue; containing opaque and birefringent particles; and granulomas. The same areas were located and analyzed on the FE-SEM and LA-ICP-MS slides. Due to sectioning across the third dimension, some areas were not present on all slides.

To date, FE-SEM analyses of 18 carbon coated samples have been conducted at $15 \mathrm{kv}$ and approximately $0.5 \mathrm{nA}$ beam current. EDS spectra (30 sec count times) of up to fifty particles, if present, from each field were acquired and phase identification made based on comparison of peak height ratios to known mineral phases. Image analysis of BSE and SE images was conducted to determine the number of particles in each field of view normalized to lung tissue area (fig. 1). LA-ICP-MS maps were generated using a $193 \mathrm{~nm}$ excimer laser ablation system coupled to a quadrupole ICP-MS. For each sample 6 or more areas $(0.5$ $\mathrm{mm}^{2}$ each) were ablated in scanning mode with $32 \mu \mathrm{m}$ laser spot size. The laser energy density was set at $1 \mathrm{~J} / \mathrm{cm}^{2}$ (below the ablation threshold of quartz) in order to only ablate the tissue sections. Data processing included gas blank, detection limit and tissue vs alveolar space correction, and normalization to sulfur for the endogenous elements (sodium, iron, zinc, phosphorus, etc.) and sum to a $100 \%$ normalization for the exogenous elements (aluminum, silicon, lead, nickel, vanadium, uranium, etc.)

The modal proportions of exogenous and endogenous phases identified among 18 samples via FESEM vary greatly. Endogenous phases include calcium phosphates, iron-rich particles, and possible silicate mineral dissolution/reprecipitation products. Exogenous phases include geologic-sourced silica, clays, feldspars, iron-titanium oxides, and barium sulfate, and some anthropogenic-sourced material such as phases containing tin, copper, zinc, mercury, nickel, lead, osmium, cobalt, and gold. The number of particles per square $\mathrm{mm}$ of tissue varied from $<10,000$ to $>50,000$. The LA-ICP-MS study showed particulate matter clusters of aluminosilicates containing titanium, vanadium, chromium, nickel, cobalt, copper, cadmium, tin, thallium, lead, thorium and uranium (fig. 2). Large differences in total amounts of 
particulate matter clusters as well as trace element composition were observed between samples from different subjects. Future directions will involve analysis to determine differences between deployer lung tissue samples compared to positive and negative controls.
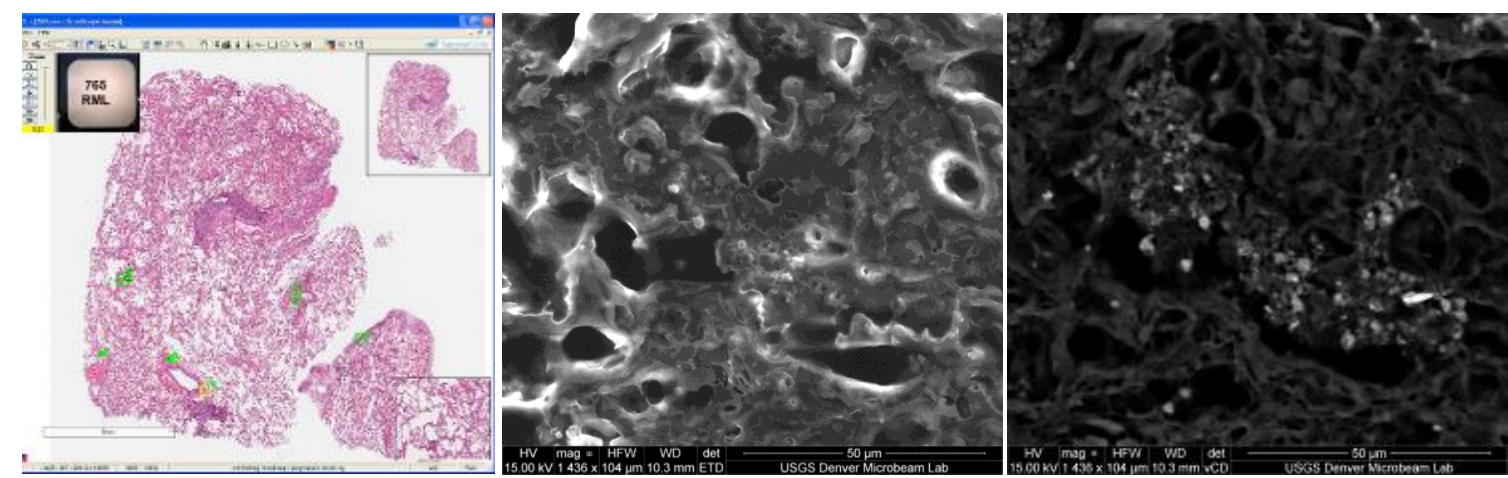

Figure 1. Areas identified on the H\&E slide (left) were located for SE (middle) and BSE (right) imaging. The number of particles present in the field of view was determined and SEM-EDS analyses of up to 50 particles in each field were acquired.

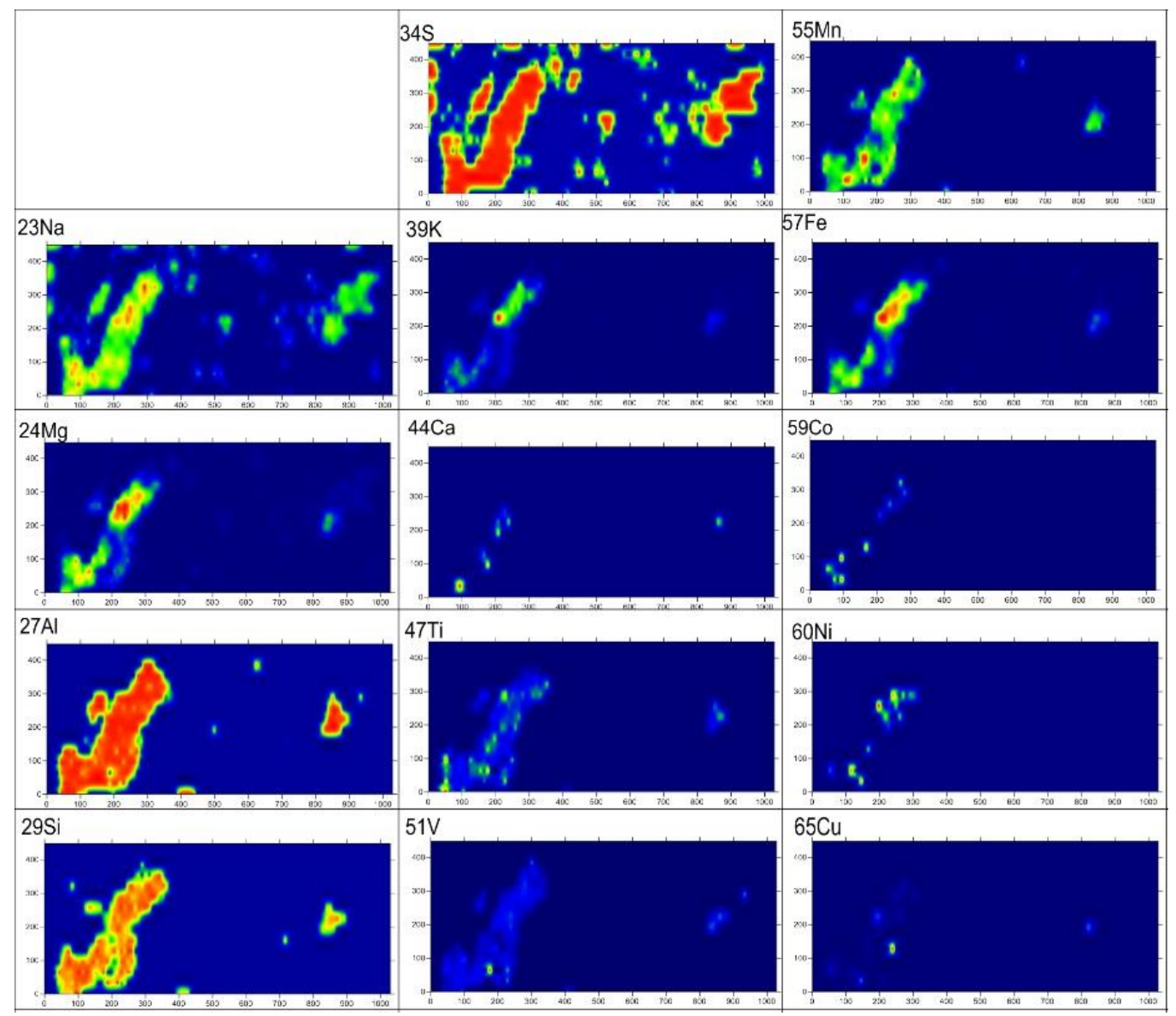

Figure 2. LA-ICP-MS distribution maps of multiple elements. 